\title{
The Growth of Catalyst-free NiO Nanowires
}

\author{
Wenhui Zhu' ${ }^{1}$, Jonathan P Winterstein ${ }^{2}$, Renu Sharma ${ }^{2}$ and Guangwen Zhou ${ }^{1}$
}

1. Department of Mechanical Engineering \& Materials Science and Engineering Program, State University of New York, Binghamton, NY, USA

2. Center for Nanoscale Science and Technology, National Institute of Standards and Technology, Gaithersburg, MD, USA

$\mathrm{NiO}$ is a stable p-type semiconductor with wide band gap $(3.74 \mathrm{eV})$. Nanostructured $\mathrm{NiO}$ has drawn much attention as a low-cost material for several applications including electrochromic devices, electrode materials in battery systems, and electrochemical supercapacitors. It is also one of the most promising materials for resistive-switching memory devices. There have been reports of different methods to prepare $\mathrm{NiO}$ nanocrystals, including evaporation, sputtering, sol-gel techniques and electrochemical deposition using anodic alumina membranes (AAM). To the best of our knowledge, there have been no reports on thermal oxidation-driven $\mathrm{NiO}$ nanowire growth. Thermal oxidation is a proven, low-cost, easy-to-control approach for growing oxide nanowires such as $\mathrm{CuO}[1], \alpha-\mathrm{Fe}_{2} \mathrm{O}_{3}[2,3]$ and $\mathrm{ZnO}$ [4]. Here we present the in situ study of $\mathrm{NiO}$ nanowire growth in an environmental transmission electron microscope (ETEM) and elucidate the atomic structure, morphology, and growth mechanism of $\mathrm{NiO}$ nanowires from the oxidation of $\mathrm{Ni}$.

A pure Ni foil was loaded in a TEM furnace heating holder followed by plasma cleaning for the in situ environmental TEM experiments. Pure oxygen $(99.9999 \%)$ was introduced into the microscope column to oxidize the $\mathrm{Ni}$ foil. After achieving a stable $\mathrm{O}_{2}$ partial pressure of $0.6 \mathrm{~Pa}$, the specimen was heated to $500{ }^{\circ} \mathrm{C}$ and held at this temperature for oxidation. Meanwhile, the oxidation driven $\mathrm{NiO}$ nanowire formation was monitored, in situ, by various TEM techniques including imaging, diffraction, and electron energy-loss spectroscopy (EELS) using the aberration-corrected ETEM operated at $300 \mathrm{kV}$.

Our in situ TEM observations show that $\mathrm{NiO}$ nanowires start to grow after 30 min of $\mathrm{O}_{2}$ flow at $500{ }^{\circ} \mathrm{C}$. Electron diffraction analysis show that the nanowires grow on a $\mathrm{NiO}$ layer, instead of directly on the $\mathrm{Ni}$ metal substrate. Fig. 1a is a TEM image of a $\mathrm{NiO}$ nanowire and Fig. 1b is a nanodiffraction pattern obtained from the nanowire, which can be indexed well with the crystal structure of $\mathrm{NiO}$ oriented along $<011>$ zone axis. Fig. 1c is an HRTEM image of the NiO nanowire, which shows clearly that the nanowire has a bicrystal structure with the $\langle 011\rangle$ zone axis and $\{111\}$ grain boundary plane. The bicrystal structure was not observed in the nanodiffraction pattern since it was collected from a small region that included only one half of the bicrystal. The diffractograms (insets in Fig. 2c) of both sides of the bicrystal, show that the two crystals have a rotation angle of about $16.5^{\circ}$. The axial growth direction of the NiO nanowire is <111>. The HRTEM image also shows that the nanowire has $\{111\}$ side facets. $\{111\}$ planes are normally the low surface-energy planes for a cubic structure such as NiO. This implies the one dimensional growth along the <111> direction is driven by the minimization of the surface energy of the nanowire. Fig. 1(d) shows the EELS obtained from the nanowire. Quantitative analysis of the $\mathrm{O} \mathrm{K}$ and $\mathrm{Ni} \mathrm{L}$ edges of the EELS gives an atomic ratio of $\mathrm{Ni}$ to $\mathrm{O}$ of $1: 1$.

Fig. 2(a-e) show a series of TEM images extracted from an in situ TEM video capturing the growth of a single $\mathrm{NiO}$ nanowire. The growth kinetics shown in Fig. $2 \mathrm{f}$ was obtained by measuring the length evolution of the nanowires from the in situ TEM video. The data were fit well with a linear model, 
which yielded a growth rate of $\approx 0.7 \mathrm{~nm} \cdot \mathrm{min}^{-1}$.

In summary, we have demonstrated that thermal oxidation of $\mathrm{Ni}$ can be employed to form $\mathrm{NiO}$ nanowires. It is shown that the $\mathrm{NiO}$ nanowires have a bicrystal structure with the axial growth direction of <111>. Our in situ TEM observations show a linear growth behavior of the NiO nanowires. Detailed in situ TEM analysis of the $\mathrm{NiO}$ nanowire growth will be presented to illustrate the atomic process of the nanowire formation and demonstrate the tunability of the nanowire growth by controlling the oxidation of $\mathrm{Ni}$.

\section{References:}

[1] Yuan, L., et al., Acta Materialia, 2011. 59(6): p. 2491-2500.

[2] Yuan, L., et al., Journal of Materials Research, 2012. 27(07): p. 1014-1021.

[3] Yuan, L., et al., Materials Science and Engineering: B, 2012. 177(3): p. 327-336.

[4] Yuan, L., et al., Journal of Crystal Growth, 2014. 390: p. 101-108.


Figure 1. (a) A NiO nanowire grown during in situ oxidation in ETEM, (b) Nanodiffraction of the nanowire in (c), with the zone axis of $\mathrm{NiO}\langle 011\rangle$. (c) Atomic structural analysis of a NiO nanowire with the zone axis $\langle 011\rangle$. The left and right inset are the diffractograms of the marked red squares. (d) EELS spectrum of the nanowire in (b), showing $\mathrm{O} \mathrm{K}$ and Ni L edge, with the Ni:O ratio of 1:1.
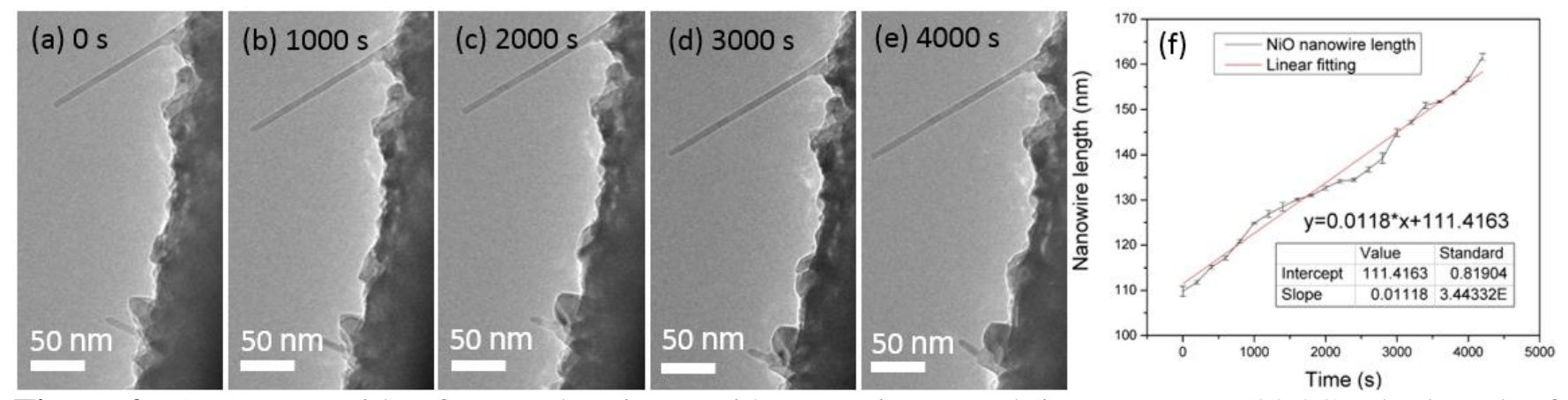

Figure 2. (a-e) TEM video frames showing a $\mathrm{NiO}$ nanowire growth in oxygen at $500{ }^{\circ} \mathrm{C}$. The length of the nanowire increased upon continued oxidation. (f) The growth rate chart of the NiO nanowire calculated by measuring the lengths of the nanowire in a series of frames in the video. After applying linear fitting, the growth rate can be determined by the slope of the fitting function, $\approx 0.0118 \mathrm{~nm} \mathrm{~s}^{-1}$ $\left(\approx 0.7 \mathrm{~nm} \cdot \mathrm{min}^{-1}\right)$ at $500{ }^{\circ} \mathrm{C}$ and oxygen partial pressure of $0.6 \mathrm{~Pa}$. 